\title{
Topography of supplementary eye field afferents to frontal eye field in macaque: Implications for mapping between saccade coordinate systems
}

\author{
JEFFREY D. SCHALL, ${ }^{1}$ ANNE MOREL, ${ }^{2}$ AND JON H. KAAS ${ }^{1}$ \\ ${ }^{1}$ Department of Psychology, Wilson Hall, Vanderbilt University, Nashville \\ ${ }^{2}$ Neurosurgery Clinic, University Hospital, Rämistrasse 100, CH-8091 Zurich, Switzerland \\ (Received July 8, 1992; ACCEPTED September 16, 1992)
}

\begin{abstract}
Two discrete areas in frontal cortex are involved in generating saccadic eye movements - the frontal eye field (FEF) and the supplementary eye field (SEF). Whereas FEF represents saccades in a topographic retinotopic map, recent evidence indicates that saccades may be represented craniotopically in SEF. To further investigate the relationship between these areas, the topographic organization of afferents to FEF from SEF in Macaca mulatta was examined by placing injections of distinct retrograde tracers into different parts of FEF that represented saccades of different amplitudes. Central FEF (lateral area 8A), which represents saccades of intermediate amplitudes, received afferents from a larger portion of SEF than did lateral FEF (area 45), which represents shorter saccades, or medial FEF (medial area 8A), which represents the longest saccades in addition to pinna movements. Moreover, in every case the zone in SEF that innervated lateral FEF (area 45) also projected to medial FEF (area 8A). In one case, a zone in rostral SEF projected to both lateral area $8 \mathrm{~A}$ from which eye movements were evoked by microstimulation as well as medial area $8 \mathrm{~A}$ from which pinna movements were elicited by microstimulation. This pattern of afferent convergence and divergence from SEF onto the retinotopic saccade map in FEF is indicative of some sort of map transformation between SEF and FEF. Such a transformation would be necessary to interconnect a topographic craniotopic saccade representation in SEF with a topographic retinotopic saccade representation in FEF.
\end{abstract}

Keywords: Saccade, Frontal eye field, Supplementary eye field, Supplementary motor area, Saccade generation, Oculomotor system, Frontal cortex

\section{Introduction}

In frontal cortex two areas are responsible for generating visually guided eye movements - the prearcuate frontal eye field (FEF) (reviewed by Goldberg \& Segraves, 1989; Bruce, 1990; Schall, 1991 $a$ ) and the dorsomedial supplementary eye field (SEF) (Schlag \& Schlag-Rey, 1987; Mann et al., 1988; Schall, $1991 b$ ). Although there are a number of functional similarities between these two regions, one pronounced difference has been identified in the nature of the saccades represented at each point within FEF and SEF. Intracortical microstimulation and single-unit recordings indicate that each point in FEF represents saccades of a particular direction and amplitude that does not vary with orbital position (Bruce \& Goldberg, 1985; Bruce et al., 1985; Segraves \& Goldberg, 1987; Schall, 1991c). Moreover, saccade amplitude is mapped in a fairly regular fashion in FEF;

Reprint requests to: Jeffrey D. Schall, Department of Psychology, Wilson Hall, Vanderbilt University, Nashville, TN 37240, USA. shorter saccades are represented ventrolaterally in the inferior limb of the arcuate sulcus, while progressively longer saccades are represented dorsomedially along the superior limb of the arcuate sulcus (Robinson \& Fuchs, 1969; Bruce et al., 1985). In contrast, microstimulation and single-unit recordings have revealed a somewhat different representation of saccades within SEF; many sites appear to represent final orbital position rather than saccade vector (Schlag \& Schlag-Rey, 1985, 1986, 1987; Schall, 1991b,c; Mitz \& Godschalk, 1989; Tehovnik \& Lee, 1990; Mann et al., 1988; Lee \& Tehovnik, 1991; but see Russo \& Bruce, 1990, 1991).

Previous anatomical studies have demonstrated that FEF and SEF are interconnected (e.g. Arikuni et al., 1988; Huerta \& Kaas, 1990; Huerta et al., 1987; Luppino et al., 1990). There is also preliminary evidence for some degree of topography in the connections between FEF and SEF (Stanton, 1986). This paper describes the first direct investigation of the topographic organization of the mapping between FEF and SEF using multiple tracer injections in the same animal. Given the orderly map- 
ping of saccade amplitude in FEF and the apparently different coordinate systems represented in FEF and SEF, it is important to understand how the two areas are interconnected. Toward that end, multiple retrograde tracers were injected into different parts of the saccade representation in FEF, and the spatial distribution of retrogradely labeled neurons in SEF was examined.

\section{Materials and methods}

Three rhesus monkeys (4-8 $\mathrm{kg}$ ) provided the data for this study. The animals were cared for according to the National Institutes of Health Guide for the Care and Use of Laboratory Animals and the guidelines of the Vanderbilt University Animal Care Committee. Placement of tracer injections in the prearcuate gyrus was guided by the results of intracortical microstimulation and surface landmarks under aseptic conditions in ketamine anesthetized monkeys. Animals were premedicated with atropine $(0.05 \mathrm{mg} / \mathrm{kg})$ and dexamethasone $(1 \mathrm{mg} / \mathrm{kg})$. Anesthesia was induced with ketamine $(30 \mathrm{mg} / \mathrm{kg})$ and maintained with periodic injections $(15 \mathrm{mg} / \mathrm{kg})$. Throughout the procedure animals were maintained on a heating pad; their temperature, respiration, and heart rate were monitored and maintained within optimal limits. A large craniotomy was made, and the underlying dura mater was retracted to expose the prearcuate gyrus. To prevent desiccation, a well of acrylic cement was built around the craniotomy and filled with silicone fluid. A stainless-steel bar was cemented to the skull to restrain the head without the stereotaxic instrument. A photograph of the exposed cortex was taken, and a magnified print was made to record and guide stimulation and injection penetrations. In two of the three animals, intracortical microstimulation was performed using tungsten microelectrodes $(0.5 \mathrm{M} \Omega$ at $1000 \mathrm{~Hz})$. Penetrations were made under visual guidance via a surgical microscope. Constant-current stimulation trains lasting 50-500 ms were used; each train consisted of symmetric biphasic square wave pulses of $0.4 \mathrm{~ms}$ total duration delivered at $250 \mathrm{~Hz}$. Currents never exceeded $150 \mu \mathrm{A}$. In each penetration, the microelectrode was advanced in 500$\mu \mathrm{m}$ steps to explore the prearcuate gyrus. If the initial exploratory current evoked an eye movement, the current level at which an eye movement could be evoked on $50 \%$ of stimulus trains was taken as the threshold current. Eye movements evoked by intracortical microstimulation were monitored visually and agreed upon by two observers.

Following exploration with microstimulation, monkeys 91-13 and $91-55$ received injections of the fluorescent dyes fast blue (FB), diamidino yellow (DY), and fluorogold (FG). In monkey 91-25 injections of horseradish peroxidase (HRP) and nuclear yellow (NY) were placed according to the sulcal pattern because it was not possible to use microstimulation. Fluorescent dyes were injected in $3 \%$ aqueous solution. WGA-HRP was injected in $2 \%$ saline. All injections were made with a 1 - or $5-\mu l$ Hamilton syringe to which a pulled glass micropipette was attached; tracers were delivered at an approximate rate of $0.05-$ $0.1 \mu \mathrm{l} / 20 \mathrm{~min} / \mathrm{mm}$. Following recovery from anesthesia, monkeys were given analgesics (butorphanol, $0.05 \mathrm{mg} / \mathrm{kg}$ or banamine, $1 \mathrm{mg} / \mathrm{kg}$ ). Animals that received only fluorescent dyes recovered for a period of 15 days before being deeply anesthetized and perfused transcardially with $0.9 \%$ saline, followed by $8 \%$ paraformaldehyde in phosphate buffer $(0.1 \mathrm{M} ; \mathrm{pH} 7.4)$ and successively with $10 \%, 20 \%$, and $30 \%$ sucrose in the same buffer. The animal that received WGA-HRP and NY survived
3 days before perfusion. The perfusion procedure was similar to that for fluorescent dye injections except that the brain was fixed with a $4 \%$ paraformaldehyde solution. After perfusion, the brains were immediately removed, photographed, and separated into two blocks by a frontal section made rostral to the central sulcus. Only the hemisphere ipsilateral to the injections was studied. The rostral blocks were sectioned coronally in two cases (91-13 and 91-25) and parasagittally in the other (91-55). Sections were $50 \mu \mathrm{m}$ thick and every third section containing fluorescent tracer was immediately mounted onto gelatinized slides, coverslipped, and stored at $4^{\circ} \mathrm{C}$. Alternate sections were stained for Nissl (cresyl violet or thionin). In 91-25, a series of sections was processed for HRP histochemistry with the low artifact tetramethyl-benzidine (TMB) method (Gibson et al., 1984). Sections containing fluorescent labeling were observed under ultraviolet illumination (355-425 nm) using a Leitz fluorescent microscope and oil-immersion objectives. FB-labeled neurons exhibited a blue cytoplasm. FG-labeled neurons exhibited the characteristic filling of the soma and proximal dendrites. DY - and NY-labeled neurons exhibited a yellow nucleus. WGAHRP labeling was observed under bright- and dark-field illumination. Cells were plotted using a computerized microscopy system. Contours of the sections and the distribution of labeled neurons were digitized via $x-y$ position encoders coupled to the microscope stage. Nissl-stained sections were then projected onto the drawings to trace contours of cortical layers, limits of sulci, and the positions of cytoarchitectonic borders. Twodimensional reconstructions of injection sites and retrogradely labeled cortex were produced using either wire models or computer graphic representations according to previously established principles (Van Essen \& Maunsell, 1980).

\section{Results}

Injections of tracers were placed in different portions of the prearcuate gyrus in three monkeys (Fig. 1). The locations of the injections were analyzed in flattened reconstructions of the arcuate cortex in relation to cytoarchitectural boundaries. We relied on previous descriptions of the cytoarchitecture of the FEF as the region of cortex along the anterior wall of the arcuate gyrus with a distinct granule cell layer and large layer $\mathrm{V}$ pyramidal cells (Stanton et al., 1989; Preuss \& Goldman-Rakic, 1991). The most lateral part of this field was further distinguished by an increase in the density of large pyramidal cells in layer III. These two portions of FEF correspond to fields $8 \mathrm{~A}$ and 45 of Walker (1940). Area 6 forms the caudal boundary of areas $8 \mathrm{~A}$ and 45 near the fundus of the arcuate sulcus. Area 45 was bounded rostrally by transitional cortex that was identified in our material as area $8 \mathrm{~A}$ (Preuss \& Goldman-Rakic, 1991). Area 46 extended from the rostral border of area $8 \mathrm{~A}$.

All injections labeled neurons in the SEF; moreover, the extent and spatial distribution of label varied in SEF according to the location of the injection in FEF. The most compelling results were obtained in case 91-13 in which extensive information about the organization of the FEF was obtained directly by microstimulation, and three different tracers were placed at three identified locations in the field. The FB injection in case 91-13 was the most lateral of the three injections placed in the animal (Figs. 1A and 1B). It was centered on the lip of the prearcuate gyrus, straddling the transition between area 45 and rostral area $8 \mathrm{~A}$; the injection extended only one-third of the way to the fundus. The core of the FB injection site included both 

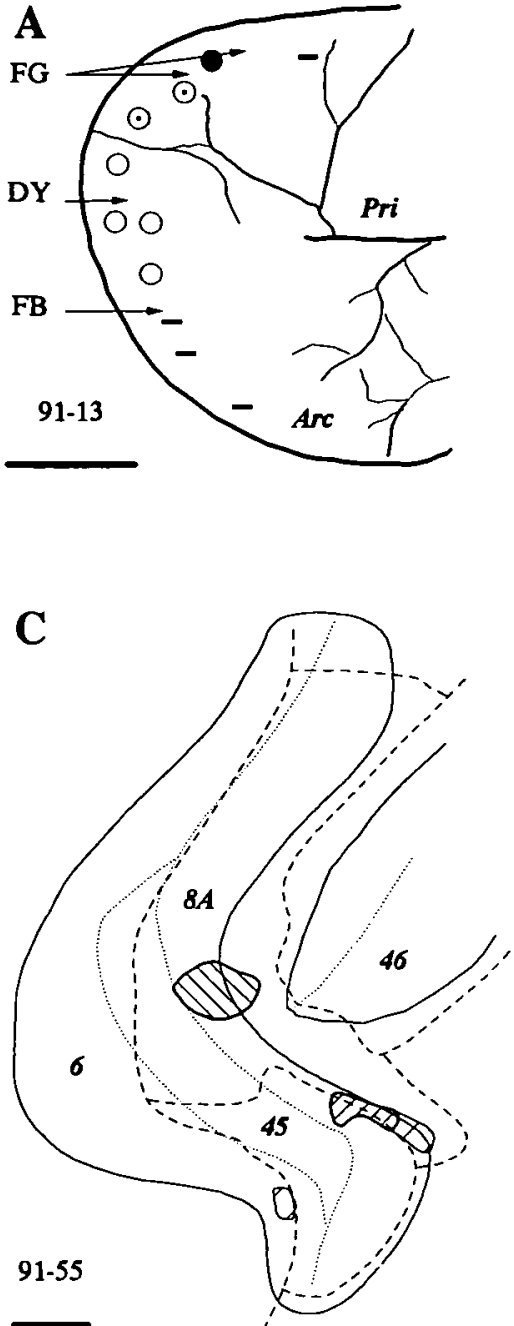

B
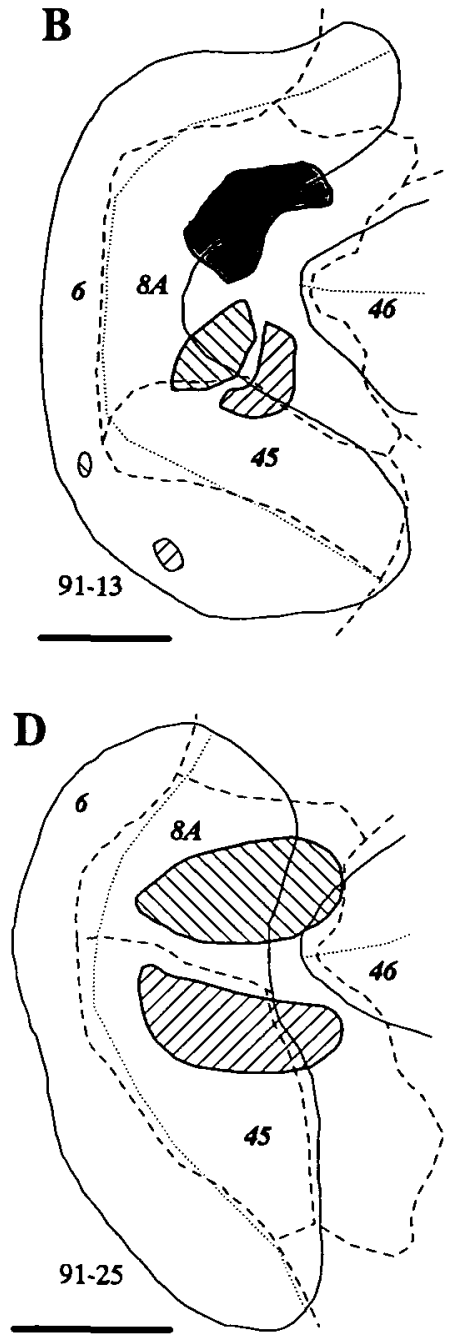

Fig. 1. Injection locations in the three cases. A: Drawing from the photograph taken during the experiment illustrating the results of microstimulation. Symbols indicate the locations of microelectrode penetrations. Ear movements $(\bullet)$ and combined eye and ear movements $(\odot)$ were evoked medially; eye movements $(0)$ alone were evoked in a middle zone, and no detectable movements (-) were evoked rostromedially and laterally. Arrows indicate the penetration locations for the labeled tracer injections. Arc: arcuate sulcus, and Pri: principal sulcus. B, C, D: Injection locations in two-dimensional reconstructions of the arcuate cortex. Solid lines indicate the lips of sulci; dotted lines show the location of fundi. Dashed lines indicate the cytoarchitectural boundaries of the labeled areas. Injection uptake zones are illustrated as hatched or shaded regions. Right hatch signifies the FB injections in 91-13 and 91-55 and the HRP injection in 91-25. Left hatch signifies the DY injection in 91-13 and 91-55 and the NY injection in 91-25. Shading signifies the FG injection in 91-13. For every panel, scale bar $=5 \mathrm{~mm}$. the most lateral sites where no clear eye movements were evoked and sites where only small eye movements were evoked. The injection was placed in this location because we reasoned that the lateral-most location of clearly evoked eye movements in the ketamine-anesthetized monkey underestimated the true lateral extent of the functional FEF. This is because ketamine has been shown to inactivate the neural integrator of the brain-stem saccade generator (Bruce \& Russo, 1987; Godaux et al., 1990) which would make detection of small saccades difficult, especially without appropriate measurement devices. The fact that the FB injection was located at the caudomedial end of area 45 is consistent with this interpretation. The injection of DY was in a more caudal medial portion of the FEF, where stimulation sites along electrode penetrations evoked clear eye movements of moderate amplitudes. The DY injection site was also located on the lip of the sulcus, adjacent to the medial border of area 45. The FB and DY injections also involved the superficial layers of part of the postarcuate gyrus; we do not think that this was reflected in the distribution patterns of labeled cells in the SEF. The medial FG injection site included the portion of the FEF where both eye and pinna movements were evoked by microstimulation. The most rostral portion of the injection core was in cortex where only ear movements were detected. This injection site was localized centrally in area $8 \mathrm{~A}$.
All three injections in 91-13 labeled dense concentrations of neurons in a delimited zone on the dorsomedial convexity (Fig. 2). Labeled neurons were observed in layers II through VI. A second focus of labeled neurons located only in the upper layers on the medial wall, caudal to the major afferent zone was contained within the supplementary motor area, probably as a result of the injections extending into the postarcuate premotor cortex. The dashed outlined area in Fig. 2 indicates the cytoarchitectural limits of SEF, corresponding to field F7 of Matelli et al. (1991), and the rostral part of 6DR (Barbas \& Pandya, 1987; Preuss \& Goldman-Rakic, 1991). In our Nissl sections, we distinguished SEF from more caudal cortex by the presence of an incipient granular layer and more distinct sublamination in layers V and VI. Rostral to SEF cortex identified as area 9 exhibited a thicker layer IV and few large layer III pyramidal cells. The medial boundary of SEF appears to be along the dorsal convexity, it being both structurally and functionally distinct from an area on the medial wall (Barbas \& Pandya, 1987; Matelli et al., 1991; Preuss \& Goldman-Rakic, 1991; Luppino et al., 1991). Consistent with these cytoarchitectural observations, the neurons retrogradely labeled by FEF injections were aligned in a remarkably sharp manner along this medial boundary. In contrast, the lateral boundary of SEF was not distinct in our material, but lateral to the dashed line in 

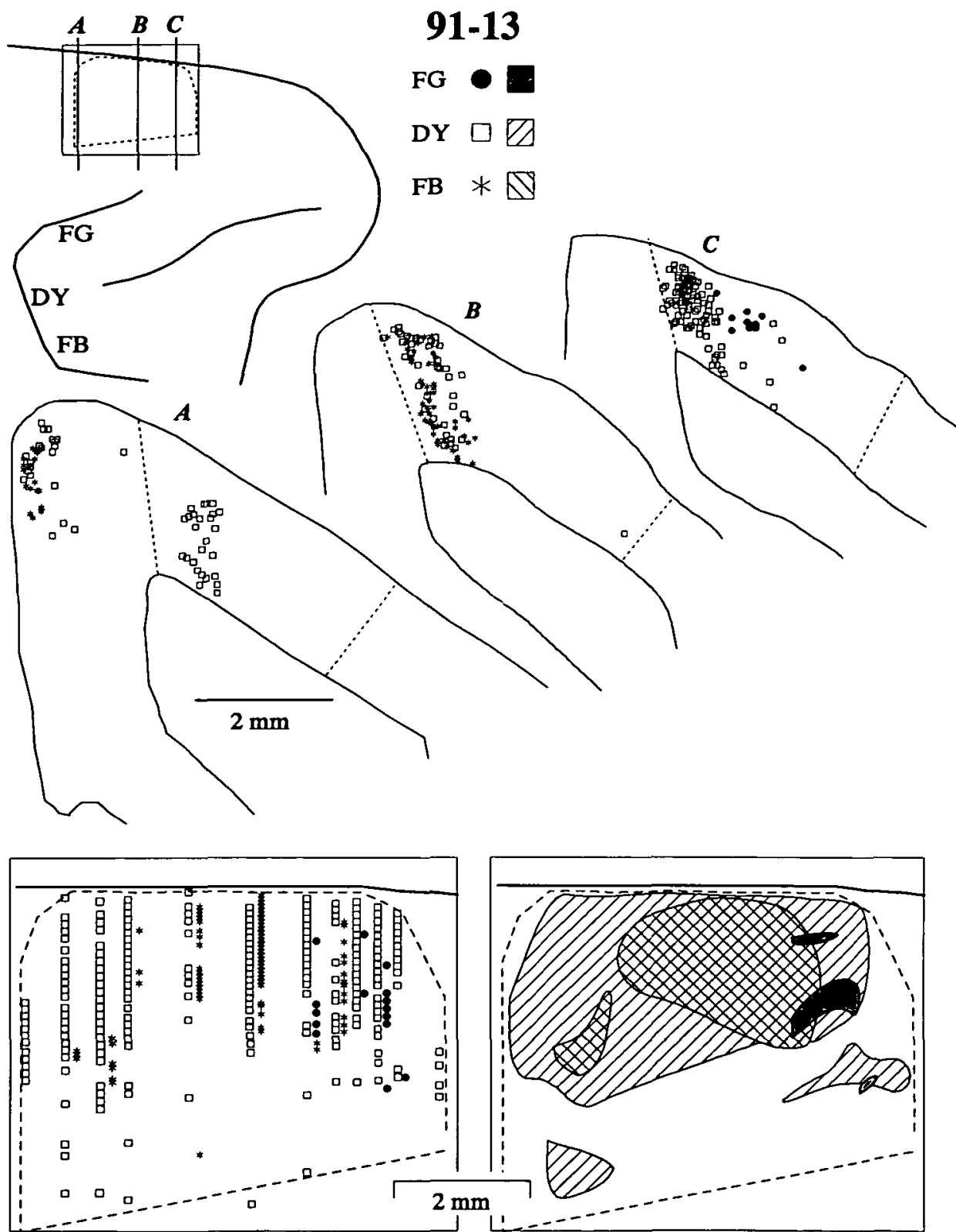

Fig. 2. The distributions of retrogradely labeled neurons in the SEF after injections of different tracers into medial, middle, and lateral locations of the FEF in monkey 91-13. The upper left diagram shows a dorsolateral view of a portion of the right frontal lobe with the arcuate and principal sulci as landmarks. The relative locations of the different tracer injections as well as the location of SEF are indicated. Portions of three coronal sections with neurons labeled by the injections are shown below; dashed lines indicate the medial and lateral boundaries of SEF. Distributions of labeled neurons in SEF are portrayed in flattened reconstructions of dorsomedial cortex from coronal sections. The midline convexity is indicated by the thicker line at the top of each panel. In the left panel, relative proportions of labeled neurons are indicated by rows of symbols corresponding to specific coronal sections. The right panel summarizes the spatial distributions of the dense label for each injection.
Fig. 2 the cortex was more distinctly laminated and had smaller pyramidal cells. Our lateral border of SEF was placed to include a scattering of labeled cells and estimates of the physiological extent of the field, but part of this cortex could be outside of SEF. The physiologically defined SEF corresponds in location to the cortical region identified according to the cytoarchitecture (Schlag \& Schlag-Ray, 1987; Schall, 1991b; Luppino et al., 1991).

The spatial range of the DY-labeled neurons was essentially coextensive with the cytoarchitecturally defined SEF. Thus, a restricted portion of the FEF received input from most if not all of SEF. The zones labeled by the other two injections were more confined. The injection placed in medial cortex that represented both eye and ear movements labeled neurons only in rostral SEF. The restricted zone of label may have been a result of FG being a less-effective tracer; however, this is probably not the case because we have observed FG labeled neurons in other topographically appropriate cortical areas, e.g. caudal auditory cortex in area Tpt (Galaburda \& Pandya, 1983) near the dorsolateral limit of the medial superior temporal visual area MST (Schall et al., 1992). The lateral FB injection also resulted in more limited label in SEF; this finding cannot be attributed to tracer effectiveness because there was abundant FB label in other appropriate regions such as TEO (Schall et al., 1992). The major focus of the labeled region was in mid-rostral SMA, but a second focus was in the caudal third of the area. The results indicate that central FEF is widely connected with the SEF, that other portions may have a more restricted input, and that while a simple topographic pattern of connections is unlikely, there may be some order to the innervation.

Additional results were obtained from monkey 91-55 in which two injections were placed in FEF (Fig. 1C). The medial injection of DY was located near the center of area $8 \mathrm{~A}$; it extended from the fundus onto the rostral lip of the arcuate sul- 
cus. The lateral injection of FB was smaller; it was located on the lip of the gyrus, straddling the transition between area 45 and rostral $8 \mathrm{~A}$. Neurons throughout the cytoarchitecturally identified SEF were labeled by the DY injection (Fig. 3), again demonstrating that central-medial FEF receives input from most of SEF. As in the previous case, the lateral border of SEF could not be positively identified, and labeled neurons diffused laterally. The lateral FB injection in FEF, in cortex presumed to be devoted to small eye movements, labeled more foci in rostromedial SEF. The smaller labeled zone was due in part to the smaller uptake zone of the FB injection in comparison to that of the other cases; nevertheless, a lateral injection in the FEF again produced a more restricted zone of label that was contained within the large zone produced by a more medial injection into FEF.

Two tracer injections were placed in the FEF of case 91-25 (Fig. 1D). HRP was injected laterally in the prearcuate gyrus; the uptake zone was fairly large but was contained primarily within area 45 , extending into area $8 \mathrm{~A}$ on the rostral lip of the gyrus. NY was injected medially; the uptake zone was also large but within area $8 \mathrm{~A}$. Unlike in the previous cases, both uptake zones extended nearly to the fundus. The NY injection re- sulted in retrogradely labeled neurons distributed over a 7-8 $\mathrm{mm}$ long, 3-4 $\mathrm{mm}$ wide region in the dorsomedial agranular cortex (Fig. 4). Once again, the entire cytoarchitecturally defined SEF was filled with cells labeled by an injection into central area 8A. The labeled neurons were observed in all layers in SEF, but lateral to SEF scattered NY labeled cells were located only in the deeper layers. The HRP injection retrogradely labeled cells forming a band along a caudal-medial to rostral-lateral axis in SEF but not extending to the rostral, medial, or lateral limits of SEF. The results from this case confirm that the part of FEF representing longer saccades receives afferents from virtually all of the cytoarchitecturally defined SEF; whereas the part of FEF representing shorter saccades receives afferents from a restricted portion of SEF that also projected to the large eye movement representation in FEF.

\section{Discussion}

This investigation provides the first direct evidence using multiple tracer injections for differential density and convergence of SEF afferents to FEF according to the amplitude of the saccades represented in FEF. Previous studies have identified con-

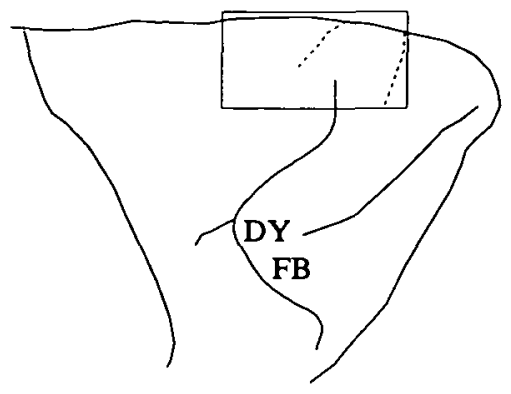

\section{1-55}

DY $\square \square$

FB $* \Delta$
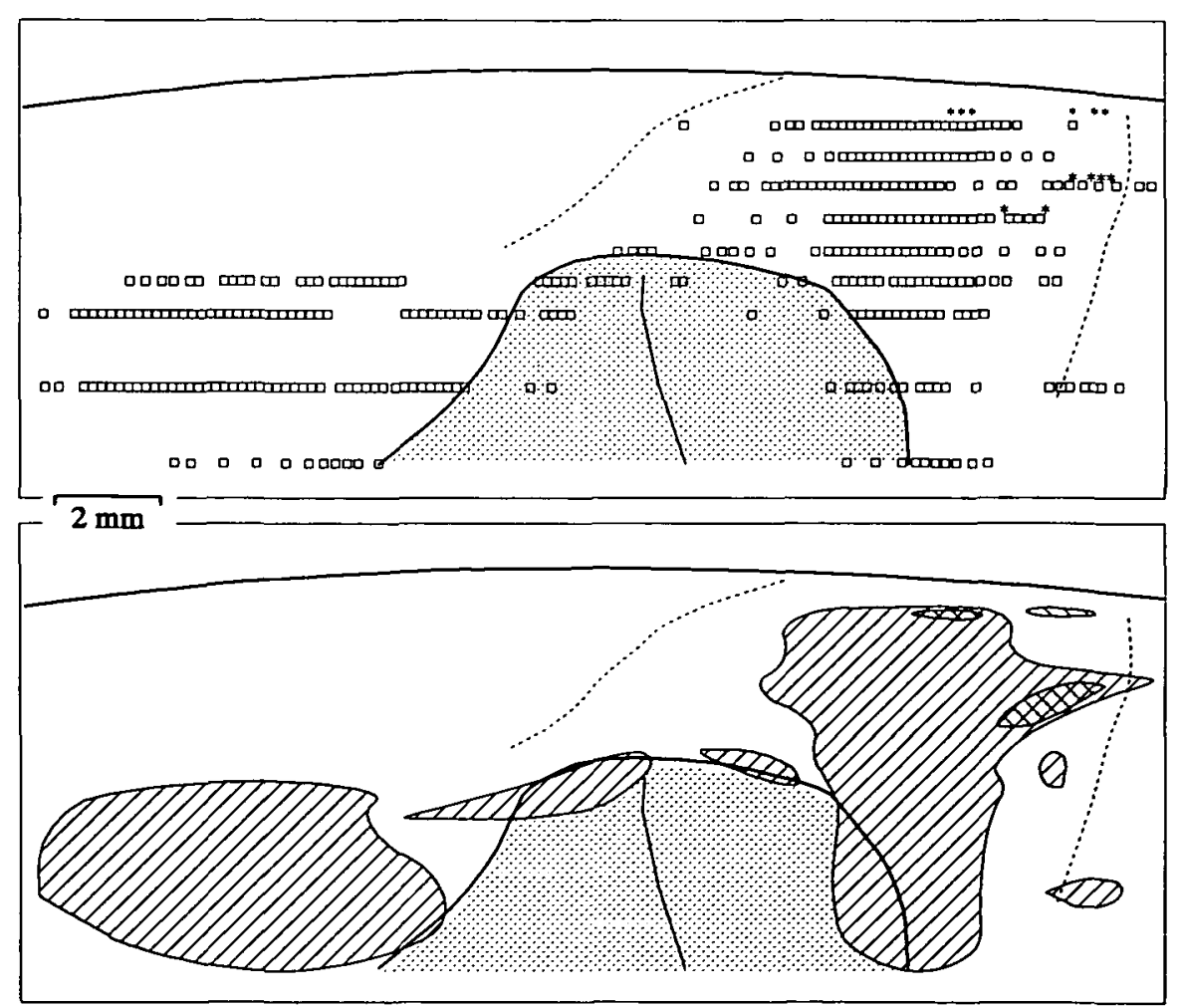

Fig. 3. Distributions of retrogradely labeled neurons in SEF after tracer injections into middle and lateral FEF in case 91-55. Conventions are as in Fig. 2 except that the sections were cut parasagittally. The shaded area in the flattened reconstructions represents the unfolded upper limb of the arcuate sulcus. In this case, the lateral boundary of SEF was not defined. 


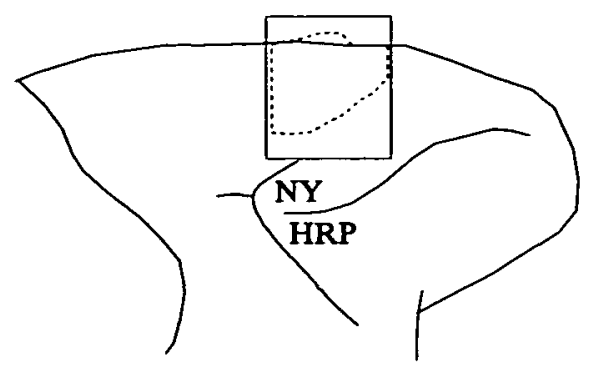

\section{1-25}

$$
\begin{gathered}
\text { NY } \square \square \\
\text { HRP } * \Delta
\end{gathered}
$$

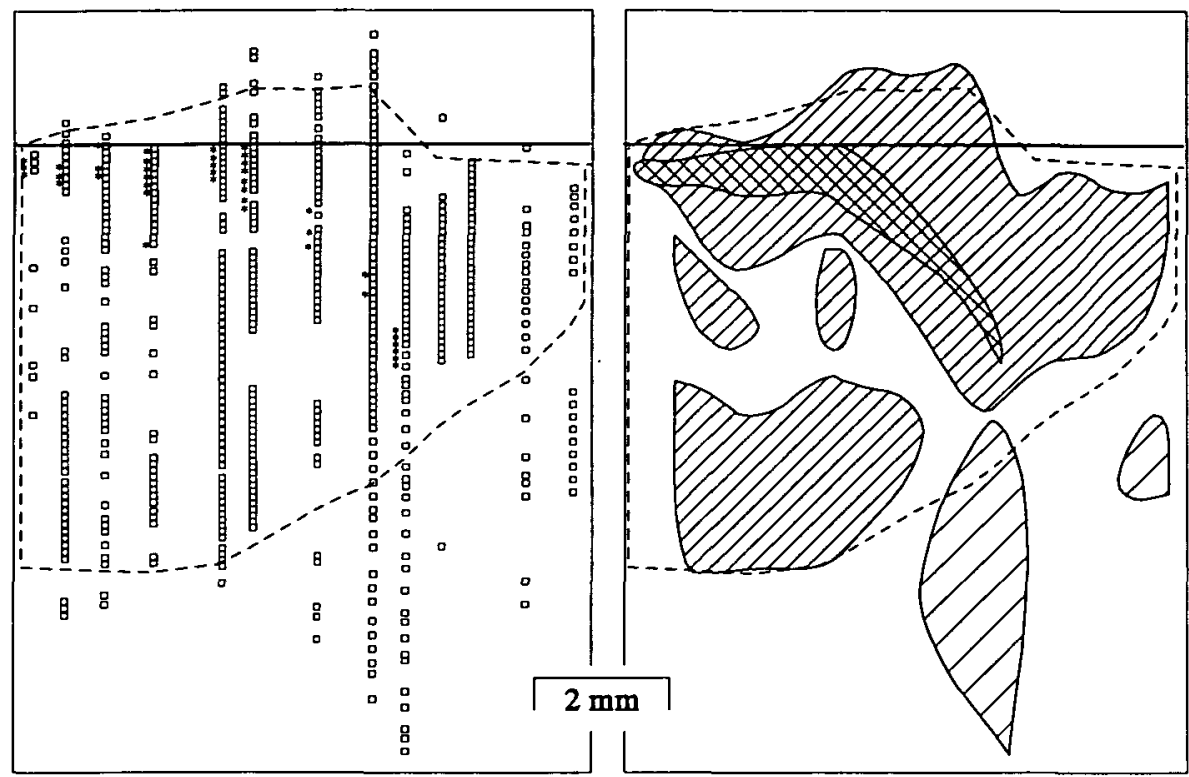

Fig. 4. Distributions of neurons labeled by tracer injections into medial and lateral FEF in monkey 91-25. Conventions are as in Fig. 2. In this animal, SEF extended somewhat onto the medial wall; this is represented by the label drawn above the midline in the flattened reconstructions. The lighter hatch indicates regions in which cells were found only infragranularly. nections between SEF and FEF (e.g. Huerta et al., 1987; Arikuni et al., 1988; Huerta \& Kaas, 1990; Luppino et al., 1990). Furthermore, the pattern of label observed in FEF following injections into SEF is consistent with the present findings (Huerta \& Kaas, 1990; Luppino et al., 1990). In particular, following a single injection in SEF, label is observed throughout the prearcuate gyrus, both in the medial, large saccade representation and in the lateral, short saccade representation. Likewise, comparing across published cases of label in dorsomedial cortex following injections into different parts of the prearcuate gyrus, the topographical relationship observed in this study can be discerned. For example, in the study by Barbas and Mesulam (1981) the most ventrolateral injection in prearcuate gyrus resulted in limited label in SEF, but the more dorsomedial injection resulted in more widely distributed label. Also, in the report of Huerta et al. (1987), an injection in dorsomedial FEF resulted in dense laterally spread label in SEF (their Fig. 4), whereas an injection situated more ventrolaterally resulted in more medially restricted label (their Fig. 5). Similar results have been reported by Arikuni et al. (1988). Finally, Stanton (1986) presented preliminary findings obtained with injections of anterograde tracers into different parts of FEF showing that lateral FEF projected to medial SEF, and medial FEF to lateral SEF.

Other findings of this study are consistent with published reports. We found that dorsomedial FEF, representing the longest saccades and pinna movements (Burman et al., 1988), received input from rostral SEF. This finding is consistent with the map of SEF published by Schlag and Schlag-Rey (1987) which shows that sites from which pinna movements could be evoked by microstimulation were rostral to the eye movement representation. The finding that parts of FEF that represent longer saccades receive input from most if not all of SEF indicates that SEF has an overrepresentation of longer saccades. This is consistent with other data in the literature; for example, Shook et al. (1990) observed that the SEF projection to the superior colliculus was more widespread and tended to be caudal to that from FEF. Thus, SEF tends to project to the parts of superior colliculus that represent larger amplitude saccades.

The data from the three cases reveal a significant degree of variability in the extent and spatial distribution of label in SEF following injections into different parts of the prearcuate gyrus. This variability can be accounted for by a combination of injection size, depth in the sulcus, and placement relative to areal borders. We were most interested in these factors as they related to the map of saccade amplitude in FEF. Comparing within and across cases, it is clear that lateral injections involving area 45 labeled fewer neurons in SEF than did medial injections involving area $8 \mathrm{~A}$. Thus, in general lateral FEF, which represents shorter saccades, received afferents from more limited parts of SEF than did medial FEF, which received afferents from most if not all of SEF. In addition, the data also indicated that the SEF input to lateral FEF arose from a medially restricted zone that was included within the larger zone projecting to medial FEF. The precise location of the portion of SEF projecting to area 45 varied somewhat in the three animals. This variation, however, may have been due to the depth of the 
injections in the prearcuate gyrus. For example, in case 91-55 the lateral, FB injection, which was the smallest of the three cases, was located on the lip of the sulcus; the labeled neurons in SEF were concentrated rostrally. Similarly, in case 91-13 the lateralmost FB injection was also centered on the lip of the gyrus but extended further toward the fundus; the labeled neurons were concentrated in a more central location within SEF. Finally, in case 91-25 the lateral, HRP injection extended from the fundus to the lip of the sulcus; the labeled neurons in SEF were found in a band extending from the caudal boundary of SEF to a point approximately two-thirds toward the rostral border.

The organization of the connections between SEF and FEF must relate to the manner in which saccades are represented in the two structures. It is well established that saccades are encoded retinotopically in FEF and that saccade amplitude is mapped systematically from ventrolateral sites representing shorter saccades to dorsomedial sites representing progressively longer saccades (Robinson \& Fuchs, 1969; Bruce et al., 1985). Saccade amplitude also varies with depth in the arcuate sulcus; larger saccades are represented near the lip and shorter saccades, near the fundus (Bruce et al., 1985). Based on this map of saccade amplitude in FEF, the pattern of label observed in SEF following injections into the different parts of the prearcuate gyrus indicates that represented saccade amplitude may increase from caudal to rostral in SEF.

A somatotopic map in the supplementary motor area with eye movements represented rostrally, surrounded by face, oral, pinna, and forelimb movements has been reported earlier (e.g. Gould et al., 1986; Schlag \& Schlag-Rey, 1987; Mitz \& Wise, 1987; Schall, 1991b; Luppino et al., 1991). More recent work has provided preliminary evidence for a topographically organized map of saccades within SEF (Tehovnik \& Lee, 1990; Lee \& Tehovnik, 1991). The nature of this topography, however, is different from that observed in FEF because of the different manner in which saccades are represented in SEF. Two lines of evidence indicate that saccades are not represented retinotopi- cally in SEF. First, microstimulation of many sites in SEF elicits saccades that vary in direction and amplitude according to initial orbital position such that gaze is brought to a specific but more or less broadly defined location (Schlag \& Schlag-Rey, 1987; Mann et al., 1988; Mitz \& Godschalk, 1989; Schall, 1991c). Second, single units have been recorded in SEF that discharge before saccades that bring the eyes to a particular orbital position, largely independent of the trajectory required to reach that position (Schlag \& Schlag-Rey, 1985, 1986; Schall, $1991 b)$. New findings indicate that the eccentricity of this goal zone varies with rostrocaudal location in SEF (Tehovnik \& Lee, 1990; Lee \& Tehovnik, 1991). In general, microstimulation of rostral sites in SEF tends to elicit saccades that converge eccentrically, directing gaze into the contralateral hemifield (see Figs. 17E and 17F of Schall, 1991c). In contrast, microstimulation of caudal sites in SEF evokes saccades that converge on a zone that is less eccentric, and if gaze is beyond that zone in the contralateral hemifield, then no saccade is evoked (see Figs. $17 \mathrm{G}$ and $17 \mathrm{H}$ of Schall, $1991 \mathrm{c}$ ). According to these data, the range of amplitudes of saccades represented at a particular site in SEF depends on the eccentricity and size of the orbital goal zone. Thus, sites that are toward rostral SEF appear to be involved in the generation of a long saccade if gaze is directed ipsilaterally as well as successively shorter saccades as gaze is shifted toward the contralateral goal zone. In contrast, sites more caudal in SEF are involved in generating a more limited range of saccades because of a less eccentric represented goal zone.

We argue that the pattern of connections observed in this study is consistent with such topographic craniotopic saccade coding in SEF. The basis of this assertion is illustrated in Fig. 5. Saccades represented by three sites within FEF and SEF and the necessary interconnections are diagrammed. In FEF saccade vector does not vary with gaze angle, but in SEF saccade vector changes with initial orbital position as illustrated. Furthermore, the three sites in SEF exhibit different patterns of orbital dependence. At one extreme, saccades bring gaze to an eccen-

\section{Frontal Eye Field Supplementary Eye Field}

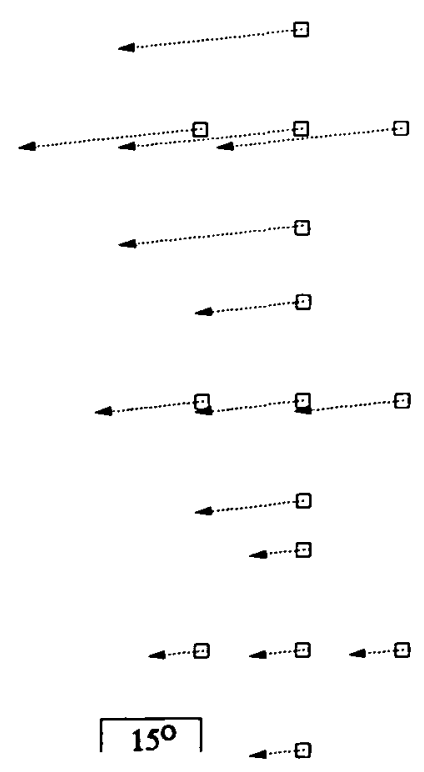

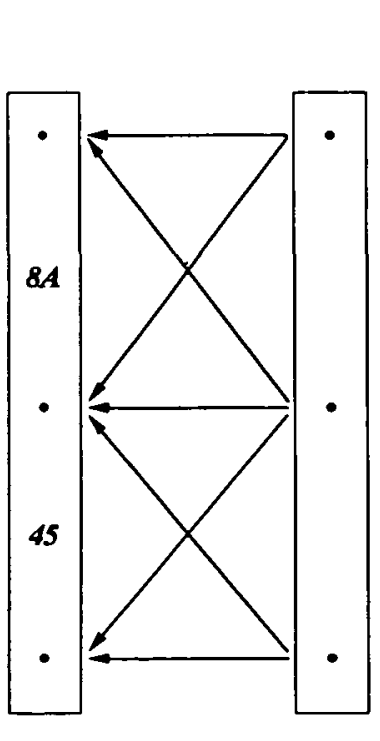

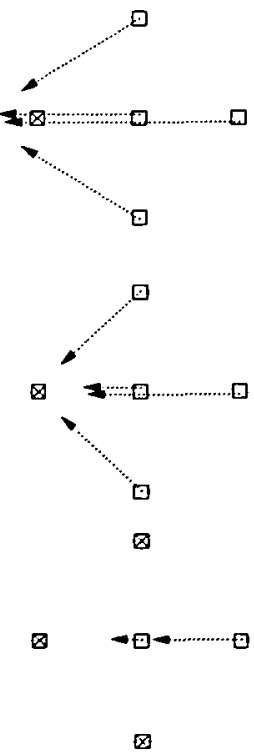

Fig. 5. Schematic connectivity between SEF and FEF saccade maps. The patterns of saccadic eye movements evoked by stimulation of three sites within each structure are illustrated; dotted arrows represent saccades elicited when the initial angle of gaze is at the locations indicated by the squares; an $x$ within a square indicates an absence of elicited saccades. The solid arrows represent the connectivity between sites representing saccades of particular amplitudes. Details are in the text. 
tric angle; if gaze is already within a certain angle of that goal region, then stimulation of SEF evokes no saccade. At the other extreme, SEF is involved in generating saccades only when gaze is in the ipsilateral visual field; thus, the resulting saccades are of more limited and less-variable amplitudes. Accordingly, the lines in Fig. 5 link sites in SEF with sites in FEF that are responsible for generating saccades of the range of amplitudes represented by a given SEF site; saccade direction is not explicitly represented. Thus, the top site in SEF is connected to the long and the intermediate saccade sites in FEF but not the short. The FEF site representing intermediate amplitude saccades is connected to all SEF sites, and the FEF site representing the shortest saccades is connected only with the bottom two SEF sites. This pattern of connectivity accounts schematically for the pattern of connectivity between SEF and FEF observed in this study as well as the body of data regarding saccade vector representation in SEF and FEF.

Evidence from well-known behavioral and neurophysiological experiments has demonstrated clearly that the position of the eye in the orbit must be accounted for by the saccade generation mechanism (e.g. Hallett \& Lightstone, 1976; Sparks \& Mays, 1983). A number of investigators have developed ideas about how the different stations of the oculomotor system can represent current eye position (e.g. Goldberg \& Bruce, 1990; Zipser \& Andersen, 1988; Andersen et al., 1990). The findings of orbital dependence of function in SEF may bear on this issue (discussed in Schall, 1991c). At present, however, not enough is known to make clear what role the observed mapping between SEF and FEF plays in saccade generation. It is clear that the pathway from SEF to FEF is not absolutely required for saccade generation. Ablation of FEF, while causing some temporary gaze deficits, does not prevent production of saccadic eye movements (e.g. Schiller et al., 1980, 1987). Moreover, following FEF ablation microstimulation of SEF is still effective in evoking saccades (Schall et al., 1987; Tehovnik et al., 1991). This result is possible because SEF projects directly to the superior colliculus and brain-stem saccade generator (Shook et al., 1990; Huerta \& Kaas, 1990). Thus, FEF and SEF may be envisaged as operating in parallel; each making some unique if as yet poorly understood contribution.

One indication of the respective roles of FEF and SEF in saccade generation is provided by the results of other ablation studies. Damage to FEF does cause permanent deficits in the generation of antisaccades in humans (Guitton et al., 1985) and of predictive saccades (Bruce \& Borden, 1986) and memoryguided saccades (Deng et al., 1986) in monkeys. Each of these deficits is expressed in individual saccades. In contrast, in humans damage to SEF causes deficits specifically in the generation of sequences of memory-guided saccades (Gaymard et al., 1990).

These clinical findings suggest another view for the role of the apparent orbital dependence observed in SEF. Normal visual inspection of an image is accomplished with fairly regular if idiosyncratic scan paths (e.g. Yarbus, 1967). Suppose that SEF functions to regulate or organize scanning patterns as opposed to single saccades. This would be consistent with the general ideas about the motor programming function of the supplementary motor area (Goldberg, 1985) and with the recent finding that certain cells in the supplementary motor area are active specifically in relation to movement sequences (Mushiake et al., 1991). For SEF to function in this capacity, each site must be involved in the generation of saccades of a range of amplitudes. For this to be the case it seems reasonable that a particular site in SEF must be connected with multiple sites in the FEF saccade map. The findings of orbital dependence of saccade generation by SEF may accordingly be a byproduct of this pattern of connectivity and not necessarily involved in representing eye position per se. This interpretation, however, then leaves open the question of what attribute of saccades is represented in SEF.

\section{Acknowledgments}

We thank Dana King, Renee Wall, Judy Ives, and Laura Trice for skilled technical assistance; Sarah Aderholt, Sarah Laborde, Frank Tu, and Christy Whalley for their help with data acquisition and analysis; and the reviewers for their helpful criticism. Supported by R01-EY08890 to J. Schall, R01-EY02686 to J. Kaas, and by P30-EY08126 to the Vanderbilt Vision Research Center. J. Schall and J. Kaas are Kennedy Center Investigators; J. Schall is also supported by the Alfred P. Sloan Foundation and the McDonnell Pew Program in Cognitive Neuroscience.

\section{References}

Andersen, R.A., Bracewell, M., Barash, S., Gnadt, J. \& Fogassi, L. (1990). Eye position effects on visual, memory, and saccaderelated activity in areas LIP and 7a of macaque. Journal of Neuroscience 10, 1176-1196.

ARIKUni, T., Watanabe, K. \& Kubota, K. (1988). Connections of area 8 with area 6 in the brain of the macaque monkey. Journal of Comparative Neurology 277, 21-40.

Barbas, H. \& Mesulam, M.-M. (1981). Organization of afferent input to subdivisions of area 8 in the rhesus monkey. Journal of Comparative Neurology 200, 407-431.

Barbas, H. \& Pandya, D.N. (1987). Architecture and frontal cortical connections of the premotor cortex (area 6) in the rhesus monkey. Journal of Comparative Neurology 256, 211-228.

BRUCE, C.J. (1990). Integration of sensory and motor signals for saccadic eye movements in the primate frontal eye fields. In Signals and Sense in Cerebral Cortex, ed. Edelman, G.M., Gall, W.E. \& Cowan, W.M., pp. 261-314. New York: John Wiley and Sons.

BRUCE, C.J. \& Borden, J.A. (1986). The primate frontal eye fields are necessary for predictive saccadic tracking. Society for Neuroscience Abstracts 12, 1086.

Bruce, C.J. \& Goldberg, M.E. (1985). Primate frontal eye fields. I. Single neurons discharging before saccades. Journal of Neurophysiology 53, 603-635.

Bruce, C.J., Goldberg, M.E., Bushnell, C. \& Stanton, G.B. (1985). Primate frontal eye fields. II. Physiological and anatomical correlates of electrically evoked eye movements. Journal of Neurophysiology 54, 714-734.

BRUCE, C.J. \& RuSso, G.S. (1987). Effects of ketamine on oculomotor function in the monkey. Society for Neuroscience Abstracts 13, 171.

Burman, D.D., Bruce, C.J. \& Russo, G.S. (1988). Pinna movements elicited by microstimulation in the prefrontal cortex of monkeys. Society for Neuroscience Abstracts 14, 208.

Deng, S.-Y., Goldberg, M.E., Segraves, M.A., Ungerleider, L.G. \& Mishrin, M. (1986). The effect of unilateral ablation of the frontal eye fields on saccadic performance in the monkey. In Adaptive Processes in Visual and Oculomotor Systems, ed. KELLER, E.L. \& ZeE, D.S., pp. 201-208. Oxford: Pergamon.

Galaburda, A.M. \& Pandya, D.N. (1983). The intrinsic architectonic and connectional organization of the superior temporal region of the rhesus monkey. Journal of Comparative Neurology 221, 169-184.

Gaymard, B., Pierrot-Deseilligny, C. \& Rivaud, S. (1990). Impairment of sequences of memory-guided saccades after supplementary motor area lesions. Annual Review of Neurology 28, 622-626.

Grbson, A.R., Hansma, D.I., Houk, J.C. \& Robinson, F.R. (1984). A sensitive low artifact TMB procedure for the demonstration of WGA-HRP in the CNS. Brain Research 298, 235-241. 
Godaux, E., Cheron, G. \& Mettens, P. (1990). Ketamine induces failure of the oculomotor neural integrator in the cat. Neuroscience Letters 116, 162-167.

GOLDBERG, G. (1985). Supplementary motor area structure and function: Review and hypotheses. Behavior and Brain Sciences 8, 567-616.

GoldberG, M.E. \& BruCE, C.J. (1990). Primate frontal eye fields. III. Maintenance of a spatially accurate saccade signal. Journal of Neurophysiology 64, 489-508.

Goldberg, M.E. \& Segraves, M.A. (1989). Visual and frontal cortices. In The Neurobiology of Saccadic Eye Movements, ed. WURTZ, R.H. \& GoldberG, M.E., pp. 283-313. New York: Elsevier.

Gould, J.H., Cusick, C.G., Pons, T.P. \& KaAs, J.H. (1986). The relationship of corpus callosum connections to electrical stimulation maps of motor, supplementary motor, and frontal eye fields in owl monkeys. Journal of Comparative Neurology 247, 297-325.

Gurtton, D., Buchtel, H.A. \& Douglas, R.M. (1985). Frontal lobe lesions in man cause difficulties in suppressing reflexive glances and in generating goal-directed saccades. Experimental Brain Research $58,455-472$.

Hallett, P.E. \& Lightstone, A.D. (1976). Saccadic eye movements towards stimuli triggered by prior saccades. Vision Research 16, 99-106.

HUERTA, M.F. \& KAAS, J.H. (1990). Supplementary eye field as defined by intracortical microstimulation: Connections in macaques. Journal of Comparative Neurology 293, 299-330.

HuERTA, M.F., Krubitzer, L.A. \& KaAs, J.H. (1987). Frontal eye fields as defined by intracortical microstimulation in squirrel monkeys, owl monkeys, and macaque monkeys. II. Cortical connections. Journal of Comparative Neurology 271, 473-492.

LEE, K.M. \& TEHOVNIK, E.J. (1991). Eye position dependency of units in the dorsomedial frontal cortex. Society for Neuroscience Abstracts $17,546$.

Luppino, G., Matelli, M. \& Rizzolatti, G. (1990). Cortico-cortical connections of two electrophysiologically identified arm representations in the mesial agranular frontal cortex. Experimental Brain Research 82, 214-218.

Luppino, G., Matelli, M., Camarda, R.M., Gallese, V. \& Rizzolatti, G. (1991). Multiple representations of body movements in mesial area 6 and the adjacent cingulate cortex: An intracortical microstimulation study in the macaque monkey. Journal of Comparative Neu. rology 311, 463-482.

Mann, S.E., Thau, R. \& Schiller, P.H. (1988). Conditional taskrelated responses in monkey dorsomedial frontal cortex. Experimental Brain Research 69, 460-468.

Matelli, M., Luppino, G. \& Rizzolatti, G. (1991). Architecture of superior and mesial area 6 and the adjacent cingulate cortex in the macaque monkey. Journal of Comparative Neurology 311, 445-462.

Mitz, A.R. \& Godschalk, M. (1989). Eye movement representation in the frontal lobe of rhesus monkeys. Neuroscience Letters 106, 157-162.

Mitz, A.R. \& WISE, S.P. (1987). The somatotopic organization of the supplementary motor area: Intracortical microstimulation mapping. Journal of Neuroscience 7, 1010-1021.

Mushiake, H., INASE, M. \& TANJI, J. (1991). Neuronal activity in the primate premotor, supplementary, and precentral motor cortex during visually guided and internally determined sequential movements. Journal of Neurophysiology 66, 705-718.

Preuss, T.M. \& Goldman-RaKic, P.S. (1991). Myelo- and cytoarchitecture of the granular frontal cortex and surrounding regions of the strepsirhine primate Galago and the anthropoid primate $\mathrm{Ma}$ caca. Journal of Comparative Neurology 310, 429-474.

Rosinson, D.A. \& Fuchs, A.F. (1969). Eye movements evoked by stimulation of frontal eye fields. Journal of Neurophysiology 32, 637-648.

Russo, G.S. \& BRuCE, C.J. (1990). Quantitative analysis of the trajectory of electrically elicited saccadic eye movements in the frontal eye field and supplementary eye field of the monkey. Society for Neuroscience Abstracts 16, 899 .

Russo, G.S. \& BRUCE, C.J. (1991). Response fields of neurons in the supplementary eye field of the rhesus monkey are retinotopic. Society for Neuroscience Abstracts 17, 462.

SCHALl, J.D. (1991a). Neural basis of saccadic eye movements in primates. In Vision and Visual Dysfunction. Vol. 4: The Neural Basis of Visual Function, ed. Leventhal, A.G., pp. 388-442. London: MacMillan Press.

Schall, J.D. $(1991 b)$. Neuronal activity related to visually guided saccadic eye movements in the supplementary motor area of rhesus monkeys. Journal of Neurophysiology 66, 530-558.

SCHALl, J.D. (1991c). Neuronal activity related to visually guided saccades in the frontal eye fields of rhesus monkeys: Comparison with supplementary eye fields. Journal of Neurophysiology 66, 559-579.

Schall, J.D., ManN, S.E. \& Schiller, P.H. (1987). Investigation of the roles of dorsomedial and ventrolateral premotor regions and the frontal eye fields in visually guided movements. Society for Neuroscience Abstracts 13, 1095.

Schall, J.D., Morel, A., King, D.J., Bullier, J. \& KaAs, J.H. (1992). Topographic organization of cortical visual afferents to frontal eye field in macaque: Functional convergence and segregation of processing streams (in preparation).

Schiller, P.H., True, S.D. \& Conway, J.L. (1980). Deficits in eye movements following frontal eye field and superior colliculus ablations. Journal of Neurophysiology 44, 1175-1189.

Schiller, P.H., SANDEll, J.H. \& MAUnSEll, J.H.R. (1987). The effect of frontal eye field and superior colliculus lesions on saccadic latencies in the rhesus monkey. Journal of Neurophysiology 57, 1033-1049.

SchlaG, J. \& Schlag-ReY, M. (1985). Eye fixation units in the supplementary eye field of monkey. Society for Neuroscience Abstracts $11,82$.

SchlaG, J. \& Schlag-Rey, M. (1986). Role of central thalamus and supplementary eye field in voluntary control of gaze in space. Bulletin of Tokyo Metropolitan Institute for Neurosciences (Suppl.) 17-31.

SCHLAG, J. \& SCHLAG-REY, M. (1987). Evidence for a supplementary eye field. Journal of Neurophysiology 57, 179-200.

Segraves, M.A. \& Goldberg, M.E. (1987). Functional properties of corticotectal neurons in the monkey's frontal eye fields. Journal of Neurophysiology 58, 1387-1419.

Shook, B.L., Schlag-Rey, M. \& Schlag, J. (1990). Primate supplementary eye field: 1 . Comparative aspects of mesencephalic and pontine connections. Journal of Comparative Neurology 301, 618-642.

SPARKS, D.L. \& MAYS, L.E. (1983). Spatial localization of saccade targets. I. Compensation for stimulation-induced perturbations in eye position. Journal of Neurophysiology 49, 45-63.

Stanton, G.B. (1986). Frontal eye-field (FEF) connections with dorsomedial area 6 in the macaque monkey. Society for Neuroscience Abstracts 12, 455.

Stanton, G.B., Deng, S.-Y., Goldberg, M.E. \& McMullen, N.T. (1989). Cytoarchitectural characteristics of the frontal eye fields in macaque monkeys. Journal of Comparative Neurology 282, 415-427.

TeHOVNIK, E.J. \& LEE, K.M. (1990). Electrical stimulation of the dorsomedial frontal cortex (DMFC) of the rhesus monkey. Society for Neuroscience Abstracts 16, 900.

Tehovnik, E.J., LeE, K.M. \& Schiller, P.H. (1991). Effect of frontal eye field or superior colliculus lesions on stimulation-evoked saccades elicited from the primate dorsomedial frontal cortex. Society for Neuroscience Abstracts 17, 458.

VAN ESSEN, D.C. \& MAUNSELl, J.H.R. (1980). Two-dimensional maps of the cerebral cortex. Journal of Comparative Neurology 191 , 255-281.

WALKER, A.E. (1940). A cytoarchitectural study of the prefrontal area of the macaque monkey. Journal of Comparative Neurology 73, 59-86.

Yarbus, A.L. (1967). Eye Movements and Vision. New York: Plenum Press.

ZIPSER, D. \& ANDERSEN, R.A. (1988). A back-propagation programmed network that simulates response properties of a subset of posterior parietal neurons. Nature 331, 679-684. 